\title{
Insulin Injection Technique is Associated with Glycemic Variability in Patients with Type 2 Diabetes
}

\author{
Lu Yuan · Fengfei Li · Ting Jing • Bo Ding • Yong Luo • \\ Rui Sun · Xiuping Wang · Hefeng Diao - Xiaofei Su - Lei Ye • \\ Jianhua Ma (iD)
}

Received: August 19, 2018 / Published online: October 19, 2018

(C) The Author(s) 2018

\begin{abstract}
Introduction: Patients with type 2 diabetes (T2D) receiving premixed insulin often fail to achieve optimal glycemic control. The insulin injection technique (IT) itself may be one of the factors affecting glycemic variability (GV). The aim of this study was to assess the relationship between GV and IT in patients with T2D using premixed insulin.

Methods: This was a single center, cross-sectional, and self-controlled trial. Patients with T2D using premixed insulin were enrolled as inpatients. The 4-day study consisted of a 2-day patient insulin injection period (days 0 and 1 ) and a 2-day specialist nurse insulin injection period (days 2 and 3). Patient insulin IT were assessed on day 1 by two independent nurses
\end{abstract}

Lu Yuan, Fengfei Li and Ting Jing contributed equally to this article.

Enhanced Digital Features To view enhanced digital features for this article go to https://doi.org/10.6084/ m9.figshare.7182269

L. Yuan · F. Li · T. Jing · B. Ding · Y. Luo - R. Sun ·

X. Wang · H. Diao $\cdot$ X. Su $\cdot$ J. Ma $(\bowtie)$

Department of Endocrinology, Nanjing First

Hospital, Nanjing Medical University, Nanjing,

China

e-mail: majianhua196503@126.com

L. Ye

National Heart Centre Singapore, National Heart

Research Institute Singapore, Singapore, Singapore using a skill-related scale consisting of 15 items, with a maximum score for each item of 2 and a total optimum score of 30 . All patients underwent 96-h continuous glucose monitoring (CGM) during the 4-day study, and CGM data collected on days 1 and 3 were recorded and analyzed. The primary outcome was the relationship between the insulin IT score and the 24-h mean amplitude glycemic excursion (MAGE) during the patient injection period.

Results: A total of 52 inpatients with T2D who used premixed insulin were recruited and completed the study. The mean total insulin IT score of these patients was considerably lower than the optimum score $(17.0 \pm 4.4$ vs. 30$)$. Our CGM data showed that the MAGE was significantly higher during the patient injection period than during the nurse injection period $(P$ $<0.05)$. Multiple linear stepwise regression analysis showed that the patient IT score was negatively correlated to the MAGE $(P<0.05)$. The patient IT score was also negatively correlated to glycated hemoglobin $\left(\mathrm{HbA}_{1 c} ; P\right.$ $<0.05)$.

Conclusions: A poorer insulin IT may negatively affect $\mathrm{GV}$ and $\mathrm{HbA}_{1 \mathrm{c}}$ control in patients with T2D using premixed insulin. Our data indicate that the insulin IT is important for short- and long-term glycemic control.

Clinical Trial Registration: ClinicalTrials.gov identification number: NCT03513055. 
Keywords: Continuous glucose monitoring; Glycemic variability; Premixed insulin injection technique; Type 2 diabetes

\section{INTRODUCTION}

The incidence of diabetes in the world has increased dramatically during recent decades, with rapid economic development, aging populations, and lifestyle changes being cited as major contributory factors [1-3]. However, glycemic control remains a tough challenge worldwide [3]. One survey conducted in China showed that only $39.7 \%$ of patients with diabetes achieved adequate glycemic control (glycated hemoglobin $\left[\mathrm{HbA}_{1 \mathrm{c}}\right]<7 \%$ ) [3], while data from the USA showed that $50.1 \%$ of diabetic patients had an $\mathrm{HbA}_{1 \mathrm{c}}$ level of $<7 \%$ [4]. Insulin is increasingly being used to manage diabetes in patients whose treatment with oral antihyperglycemic agents has failed. About $61.5 \%$ of Chinese patients with type 2 diabetes (T2D) are on insulin therapy [5]. Different from Western countries, premixed insulin is the common choice as the starter insulin preparation for Asian patients $[2,6,7]$. A retrospective database analysis of insulin therapy in Chinese patients with T2D revealed that premixed insulin was prescribed much more $(77.3 \%)$ than basal $(11.8 \%)$ and prandial (10.9\%) insulin [8]. Various studies have found that an inappropriate insulin injection technique (IT) when using the insulin pen, such as incorrect rotation of the injection site and needle reuse, may affect the efficacy of insulin therapy, eventually leading to poor glucose control $[9,10]$. Huang et al. further highlighted that the patients in their study on premixed insulin therapy failed to achieve optimal $\mathrm{HbA}_{1 \mathrm{c}}$ control [11]. In a Turkish study, about $40 \%$ of patients using an insulin injector device had glycemic variability (GV) [12].

$\mathrm{GV}$ is associated with micro- and macrovascular complications [13]. For example, the level of circulating cytokines are higher under conditions of GV than when elevated glucose concentrations are stable [14]. Continuous glucose monitoring (CGM), with its capability of recording blood glucose fluctuations, represents a new tool for studying the influence of factors on GV in real life [15].

We have conducted a single-center, crosssectional, self-controlled study to determine the relationship between GV and insulin IT in patients with T2D who used premixed insulin.

\section{METHODS}

This was a single-center, cross-sectional, selfcontrolled trial (ClinicalTrials.gov identification number: NCT03513055). The study protocol and patient consent forms were approved by the Institutional Ethical Committee of Nanjing First Hospital, Nanjing Medical University. All procedures followed were in accordance with the ethical standards of Nanjing First Hospital and with the 1964 Helsinki Declaration and its later amendments or comparable ethical standards. Informed consent was obtained from all participants included in the study.

Between May and July 2018, patients with T2D who used premixed insulin were enrolled as inpatients in the Department of Endocrinology, Nanjing First Hospital, Nanjing Medical University, China. All patients had been referred to the department by endocrinologists, and no patient came from a primary care center. The inclusion criteria were: (1) diagnosis of T2D according to World Health Organization criteria; (2) age between 18 and 80 years; (3) insulin delivered by insulin pen; (4) use of stable premixed insulin dose for at least 3 months; (5) patient willingness to undergo CGM; (6) patient self-injected insulin. Patients were excluded from the study if they met any one of the following conditions: (1) insulin was injected by a third party; (2) pregnancy, breastfeeding, or plans to become pregnant; (3) presence of acute metabolic diabetic complications, such as ketoacidosis or hyperosmolar state (coma); (4) presence of serious complications, such as cardiovascular disease, end stage renal disease, among others; (5) poor compliance and irregular eating and exercise; (6) presence of severe retinopathy or other eye problems that impair visual function; (6) cognitive dysfunction. All patients had received insulin injection 
education when they were initially put on insulin therapy.

The study included a 2-day patient insulin injection period and a 2-day specialist nurse insulin injection period (Fig. 1). The demographic data for each patient were recorded, and fasting plasma serum was collected for the measurement of $\mathrm{HbA}_{1 \mathrm{c}}$ concentration on day 0 . On days 0 and 1 , patients injected themselves with their usual dose of premixed insulin, and the IT of each patient was assessed independently by two specialist nurses who used a scale composed of 15 skill-related items according to the New Insulin Delivery Recommendations and previous studies [16-19]. Each item was weighted with a score ranging from 0 to 2 points (Table 1 ), and the scores were averaged for further analysis. On days 2 and 3, the same type of insulin (unopened) was injected by nurses with the doses unchanged. During the study period, patients received premixed insulin twice daily, once before breakfast (0700 hours) and once before dinner (1700 hours). The abdomen was used for all injection sites. In this study, all patients received insulin with 6-mm pen needle.

CGM was performed for $96 \mathrm{~h}$ on all patients during the 4 days of the study using a Medtronic Inc. (Northridge, CA, USA) CGM system, as previously described $[20,21]$. In addition, all patients were instructed to perform self-monitored blood glucose measurements, using an OneTouch Ultra Vue blood glucose monitor (LifeScan, Milpitas, CA, USA) at four time points per 24-h period: before breakfast, lunch, and dinner and before going to bed. After the CGM data were collected, the mean amplitude of glycemic excursion (MAGE) was calculated manually for each patient by measuring the arithmetic mean of the ascending and descending glycemic excursions between consecutive peaks and nadirs for the same 24-h period, and only absolute glycemic excursion values with a standard deviation (SD) of $>1$ were considered, as previously described [20-22]. Other glucose indicators were also recorded, including the 24-h mean glucose concentration (MG), the 24-h SD of the MG $(\mathrm{SD})$, the incremental area under the curve (AUC) of plasma glucose $>10.0 \mathrm{mmol} / \mathrm{L}$ and $<3.9 \mathrm{mmol} / \mathrm{L}$, and the percentage of time in that the patient was in hypo-, normo- and hyperglycemia, respectively,. The primary outcome was the relationship between the insulin IT and MAGE. The secondary outcomes were the differences in glycemic profiles according to who injected the insulin (patient vs. nurse).

\section{Statistical Analysis}

The analyses were performed using the SPSS version 16.0 statistical package (IBM Corp., Armonk, NY, USA). All variables were tested for normal distribution of the data. Data were presented as the mean $\pm \mathrm{SD}$ or as the median with the interquartile range (IQR; 25-75th percentile) according to whether the data were normally distributed or not. Square root transformation of the data was performed if the new transformed data achieved a normal distribution. The paired $t$ test was used to compare two groups when the data were normally distributed; otherwise, Wilcoxon analysis was performed. Multiple linear stepwise regression

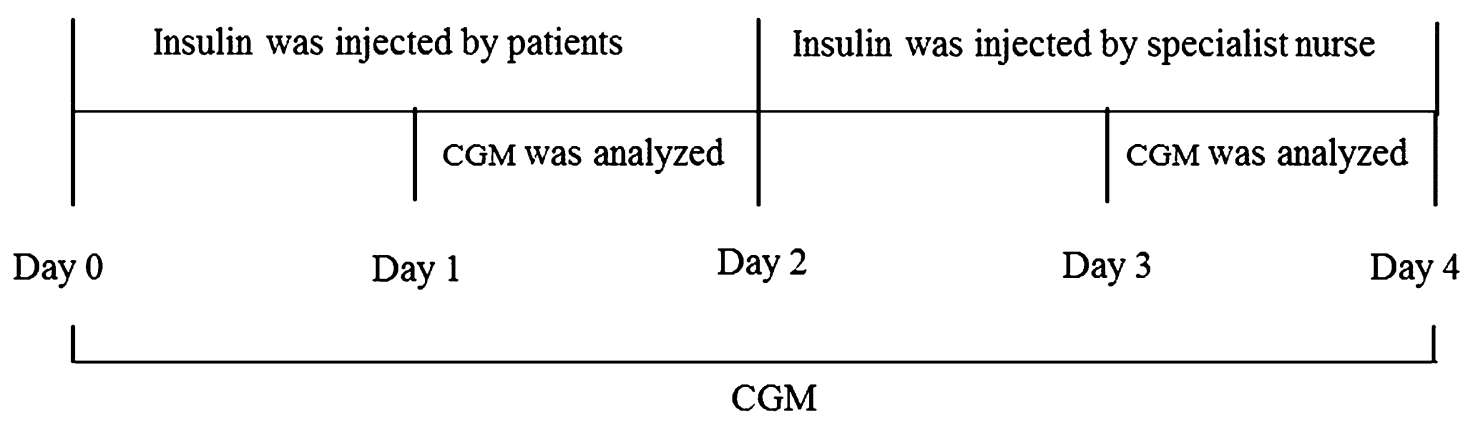

Fig. 1 Flow chart of the study. CGM Continuous glucose monitoring 
Table 1 Items and scores of the premixed insulin injection technique

\begin{tabular}{lr}
\hline Items & Scorc \\
\hline Injection at indicated time & 2 \\
Yes & 0 \\
No & 0
\end{tabular}

Scores

Warming insulin up to room temperature before injection

Yes

$>30 \mathrm{~min}$

$<30 \mathrm{~min}$

No

Checking insulin (dosage and liquid) before injection

Yes

Partly

No

Resuspension of insulin before injection

Yes

Correct

Incorrect

No

Attaching a pen needle

Correct

Incorrect

Priming before injection

Yes

No

Inspection of injection sites

Yes

No

Rotation of injection sites

Correct

Incorrect

Disinfecting the skin and injecting insulin after area of disinfection has dried

Yes

Correct

Incorrect 
Table 1 continued

\begin{tabular}{|c|c|}
\hline Items & Scores \\
\hline No & 0 \\
\hline \multicolumn{2}{|l|}{ Pinching the skin } \\
\hline Yes & 2 \\
\hline No & 0 \\
\hline \multicolumn{2}{|l|}{ Needle entry angle } \\
\hline Correct & 2 \\
\hline Incorrect & 0 \\
\hline \multicolumn{2}{|c|}{ The duration of time(s) the pen needle is under the skin } \\
\hline$>10$ & 2 \\
\hline $5-10$ & 1 \\
\hline$<5$ & 0 \\
\hline \multicolumn{2}{|c|}{ Pulling needle out while holding dose knob } \\
\hline Yes & 2 \\
\hline No & 0 \\
\hline Needle reuse (times) & 2 \\
\hline 1 & 2 \\
\hline $2-5$ & 1 \\
\hline$>5$ & 0 \\
\hline \multicolumn{2}{|c|}{ Insulin (opened and unopened) storage } \\
\hline Yes & 2 \\
\hline Partly & 1 \\
\hline No & 0 \\
\hline Maximum score of all 15 items & 30 \\
\hline
\end{tabular}

Each item was weighted with a score ranging from 0 (minimum) to 2 (maximum) points

analysis was performed to identify parameters likely to correlation with MAGE or $\mathrm{HbA}_{1 \mathrm{c}}$. Spearman's analysis was performed to identify correlation between each injection item and MAGE or $\mathrm{HbA}_{1 \mathrm{c}}$. All comparisons were twosided at the $5 \%$ significance level, and a $P$ value of $<0.05$ was considered to be statistically significant.

\section{RESULTS}

A total of 60 patients were initially recruited to this study, of whom two were excluded due to not meeting the self-injection criterion, one was excluded due to the development of sudden deafness which necessitated glucocorticoid therapy, and five were excluded due to missing 
CGM data. Thus, the data of the 52 patients (27 males, 25 females) who completed the trial were analyzed at the endpoint. The mean age of these 52 patients was $63.8 \pm 9.5$ years, their mean $\mathrm{HbA}_{1 \mathrm{c}}$ level was $8.1 \pm 1.5 \%$, and the mean body mass index (BMI) was $24.9 \pm 3.2 \mathrm{~kg} /$ $\mathrm{m}^{2}$. All patients had diabetes for a long period of time $(159.9 \pm 77.6$ months $)$, and all had used insulin for a mean of 60 (IQR 36-120) months. The mean daily insulin dose was $0.6 \pm 0.2$ $\mathrm{U} / \mathrm{kg} /$ day.

The mean total patient IT score for the 15 skill-related items was lower than the optimum score $(17.0 \pm 4.4$ vs. 30$)$. Our CGM data showed that the glucose profiles, such as MAGE, SD, $\mathrm{MG}$, and AUC $>10 \mathrm{mmol} / \mathrm{L}$, and percentage of time in a hyperglycemic state were higher during the patient injection period than during the nurse injection period $(P<0.05$, respectively). The percentage of time in the normoglycemic state in the patient injection period was significantly lower than that in the nurse injection period $(P<0.05)$. The AUC $<3.9 \mathrm{mmol} / \mathrm{L}$ and percentage of time in the hypoglycemic state were not significantly different between the two injection periods (Table 2 ).

To determine the relationship between insulin IT and the glycemic variability, we performed a multiple linear stepwise regression analysis, controlling for gender, age, BMI, $\mathrm{HbA}_{1 \mathrm{c}}$, duration of insulin therapy, dosage of insulin used per day, duration of diabetes, and insulin IT score. Our data showed that the patient insulin IT score was significantly correlated with MAGE $(B=-0.29, \quad P<0.05)$. Spearman's analysis showed that MAGE was correlated to the IT score for the items resuspension, pinching the skin, and needle reuse $(r=-0.48,-0.35$, and -0.47 , respectively; all $P<0.05)$.

We also assessed the relationship between insulin IT and long-run glycemic control. Multiple linear stepwise regression analysis, controlled for gender, age, BMI, duration of insulin therapy, dosage of insulin used per day, duration of diabetes, and insulin IT score, showed that only patient insulin IT score was significantly correlated with $\mathrm{HbA}_{1 \mathrm{c}}(B=-0.12, P$ $<0.05)$. Spearman's analysis showed that $\mathrm{HbA}_{1 \mathrm{c}}$ was negatively correlated to the score for the rotation of injection sites $(r=-0.33, P$ $<0.05)$.

\section{DISCUSSION}

In this study, we found that only $27 \%$ of patients with T2D who received premixed insulin therapy achieved the desired $\mathrm{HbA}_{1 \mathrm{c}}$ target of $\leq 7 \%$. The CGM data showed that the glucose profiles, such as MAGE, SD, MG, AUC $>10 \mathrm{mmol} / \mathrm{L}$, and percentage of time in the hyperglycemic state, were significantly higher during the patient injection period than during the nurse injection period. The percentage of time in the normoglycemic state was significantly lower in the patient injection period than in the nurse injection period. Moreover, we found that both $\mathrm{HbA}_{1 \mathrm{c}}$ level and MAGE were negatively corrected to the insulin IT score, indicating that the insulin IT may play a role in short- and long-term glycemic control.

Diabetes patients worldwide commonly use an incorrect insulin IT, such as incorrect rotation of the injection site, incorrect needle reuse, incorrect (or no) resuspension procedure, among others $[9,10]$. It is recommended that the vial or pen containing NPH (Neutral Protamine Hagedorn) insulin be tipped back and forth at least 20 times to resuspend the insulin, but few patients do this correctly, partly because the procedure is considered to be boring and unnecessary $[18,23]$. However, resuspension of the insulin is important because an incorrect resuspension may result in the delivery of an inappropriate insulin dose [24-26], which may further alter the pharmacokinetics and pharmacodynamics of insulin, possibly leading to increases in daily GV [27]. It has been reported that resuspension training can decrease hypoglycemia [23] and the $\mathrm{HbA}_{1 \mathrm{c}}$ value [18]. Only five patients in our study resuspended the premixed insulin correctly. In this context, it should be noted that Spearman's analysis of our data showed that the resuspension score of our patients was negatively correlated to MAGE as determined by CGM, indicating that the correct resuspension procedure may decrease $\mathrm{GV}$ in patients with T2D who are receiving premixed insulin. 
Table 2 The glucose profiles in the different injection periods

\begin{tabular}{llll}
\hline Parameters & Patient injection period & Nurse injection period & P value \\
\hline MG $(\mathrm{mmol} / \mathrm{L})$ & $9.5 \pm 2.2$ & $8.3 \pm 1.9$ & 0.00 \\
SD $(\mathrm{mmol} / \mathrm{L})$ & $2.2 \pm 1.0$ & $2.0 \pm 0.9$ & 0.03 \\
MAGE $(\mathrm{mmol} / \mathrm{L})$ & $5.4(3.7,7.9)$ & $3.8(2.8,5.4)$ & 0.00 \\
AUC $>10 \mathrm{mmol} / \mathrm{L}(\mathrm{mmol} / \mathrm{L} / \mathrm{day})^{\mathrm{a}}$ & $0.7(0.1,1.7)$ & $0.1(0.0,1.0)$ & 0.00 \\
AUC $<3.9 \mathrm{mmol} / \mathrm{L}(\mathrm{mmol} / \mathrm{L} / \mathrm{day})^{\mathrm{b}}$ & $0.0(0.0,0.0)$ & $0.0(0.0,0.0)$ & 0.13 \\
Percentage of time in hyperglycemia $(\%)^{\mathrm{c}}$ & $30.7(9.8,53.8)$ & $13.7(2.3,37.1)$ & 0.00 \\
${\text { Percentage of time in normoglycemia }(\%)^{\mathrm{d}}}$ & $69.3(46.2,88.2)$ & $86.1(61.5,95.1)$ & 0.00 \\
Percentage of time in hypoglycemia $(\%)^{\mathrm{e}}$ & $0.0(0.0,0.0)$ & $0.0(0.0,0.0)$ & 0.10 \\
\hline
\end{tabular}

Data are presented as mean \pm standard deviation $(\mathrm{SD})$ or as the median with the interquartile range (IQR; (25th, 75th percentile) in parenthesis

$M G$ 24-h mean glucose concentration, $S D$ standard deviation of MG, $M A G E$ 24-h mean amplitude of glycemic excursion, $A U C$ area under the curve

a AUC $>10 \mathrm{mmol} / \mathrm{L}$ is the incremental area under the curve of plasma glucose $>10.0 \mathrm{mmol} / \mathrm{L}$

b AUC $<3.9 \mathrm{mmol} / \mathrm{L}$ is the incremental area under curve of plasma glucose $<3.9 \mathrm{mmol} / \mathrm{L}$

c Percentage of time in hyperglycemia is the percentage of time the patient is is a hyperglycemic state (glucose level $>$ $10 \mathrm{mmol} / \mathrm{L}$ )

d Percentage of time in normoglycemia is the percentage of time the patient is in a normoglycemic state (glucose level from 3.9 to $10 \mathrm{mmol} / \mathrm{L}$ )

${ }^{\mathrm{e}}$ Percentage of time in hypoglycemia is the percentage of time the patient is in a hypoglycemic state (glucose level $<$ $3.9 \mathrm{mmol} / \mathrm{L}$ )

Approximately half of diabetic patients worldwide use their needles more than once [9], a practice which is associated with unexplained hypoglycemia, hyperglycemia, increased GV, and slightly higher $\mathrm{HbA}_{1 \mathrm{c}}$ levels [10]. More recently, Ji and Lou confirmed a significant relationship between needle reuse and lipohypertrophy (LH) [17]. Authors of other studies have also reported that patients with LH had significantly higher GV (defined through selfmonitoring of blood glucose) [17, 28, 29]. In our study, only $5.8 \%$ patients used their needles only once. Moreover, we identified that the needle reuse score was linked to MAGE.

Incorrect rotation of the injection site can result in $\mathrm{LH}$, which may in turn result in an increase in the $\mathrm{HbA}_{1 \mathrm{c}}$ level in diabetic patients, in both Western and Chinese diabetic populations $[9,10,30,31]$. In accordance with previous studies, our data showed that the score for the rotation of injection site was negatively correlated with the $\mathrm{HbA}_{1 \mathrm{c}}$ value.

In their worldwide study of injection practices, Frid et al. found that $63.7 \%$ of patients lifted a skinfold and that $75.0 \%$ of these did it correctly [9]. The great majority of patients in our study pinched the skin correctly. We found that the IT score for pinching the skin was negatively related to MAGE.

In this study, the total patient score for the insulin IT was calculated by summing the patient's score for each item. Analysis of the IT score revealed that both the $\mathrm{HbA}_{1 \mathrm{c}}$ level and MAGE were negatively correlated to the IT score.

The strength of our study is that we determined that correct insulin IT practices, such as resuspension of the insulin, pinching the skin, and rotation of the injection site, are important for achieving glycemic control in patients receiving premixed insulin therapy. These 
results indicate that physicians and diabetes educators should pay more attention to developing and implementing programs aimed at educating diabetes patients on their insulin IT.

The present study has several limitations. First, it was a small-scale hospital-based observation study, and there was no follow-up study. Second, we did not observe an association between insulin IT and hypoglycemia; a followup study should be performed in the future to examine the relationship between insulin IT and hypoglycemia. Third, the study was performed in Nanjing, China and involved Chinese patients only; care should be taken in extrapolating the results to diabetes patient populations in other countries.

\section{CONCLUSIONS}

Insulin IT was negatively correlated to shortand long-term glycemic control in patients with $\mathrm{T} 2 \mathrm{D}$ receiving premixed insulin.

\section{ACKNOWLEDGEMENTS}

We thank the participants of the study. We extend our appreciation to Prof. Frank Elliott who contributed to the final version of the manuscript.

Funding. This study and article processing charges were funded by Science and Technology Support Program of Jiangsu Province (CN) (No. BL2014010).

Authorship. All the named authors meet the International Committee of Medical Journal Editors (ICMJE) criteria for authorship for this article, take responsibility for the integrity of the work as a whole, and have given their approval for this version to be published.

Authorship Contributions. JHM, and LY contributed to the conception and design of the study. LY, TJ, BD, YL, RS, XPW, HFD, and XFS conducted the study and collected data. LY contributed to the data analysis. LY, and FFL wrote and gave final approval to the manuscript.

Disclosures. Lu Yuan, Fengfei Li, Ting Jing, Bo Ding, Yong Luo, Rui Sun, Xiuping Wang, Hefeng Diao, Xiaofei Su, Lei Ye, and Jianhua Ma declare that they have no conflict of interest.

Compliance with Ethics Guidelines. All procedures performed in studies involving human participants were in accordance with the ethical standards of the institutional and/or national research committee and with the 1964 Helsinki Declaration and its later amendments or comparable ethical standards. Informed consent was obtained from all participants included in the study.

Data Availability. The datasets analyzed for the current analysis are available from the corresponding author on reasonable request.

Open Access. This article is distributed under the terms of the Creative Commons Attribution-NonCommercial 4.0 International License (http://creativecommons.org/licenses/ by-nc/4.0/), which permits any noncommercial use, distribution, and reproduction in any medium, provided you give appropriate credit to the original author(s) and the source, provide a link to the Creative Commons license, and indicate if changes were made.

\section{REFERENCES}

1. Cho NH, Shaw JE, Karuranga S, et al. IDF diabetes atlas: global estimates of diabetes prevalence for 2017 and projections for 2045. Diabetes Res Clin Pract. 2018;138:271-81.

2. Yang W, Lu J, Weng J, et al. Prevalence of diabetes among men and women in China. N Engl J Med. 2010;362(12):1090-101.

3. $\mathrm{Xu} \mathrm{Y}$, Wang $\mathrm{L}, \mathrm{He}$ J, et al. Prevalence and control of diabetes in Chinese adults. JAMA. 2013;310(9):948-59.

4. Suh DC, Choi IS, Plauschinat C, Kwon J, Baron M. Impact of comorbid conditions and race/ethnicity on glycemic control among the US population with 
type 2 diabetes, $1988-1994$ to $1999-2004$. J Diabetes Complicat. 2010;24(6):382-91.

5. Guo XH, Yuan L, Lou QQ, et al. A nationwide survey of diabetes education, self-management and glycemic control in patients with type 2 diabetes in China. Chin Med J (Engl). 2012;125(23):4175-80.

6. Inzucchi SE, Bergenstal RM, Buse JB, et al. Management of hyperglycemia in type 2 diabetes: a patient-centered approach: position statement of the American Diabetes Association (ADA) and the European Association for the Study of Diabetes (EASD). Diabetes Care. 2012;35(6):1364-79.

7. Qiao Q, Nakagami T, Tuomilehto J, et al. Comparison of the fasting and the 2-h glucose criteria for diabetes in different Asian cohorts. Diabetologia. 2000;43(12):1470-5.

8. He $\mathrm{X}$, Chen $\mathrm{L}$, Wang $\mathrm{K}, \mathrm{Wu} \mathrm{H}, \mathrm{Wu}$ J. Insulin adherence and persistence among Chinese patients with type 2 diabetes: a retrospective database analysis. Patient Prefer Adherence. 2017;11:237-45.

9. Frid AH, Hirsch LJ, Menchior AR, Morel DR, Strauss KW. Worldwide injection technique questionnaire study: population parameters and injection practices. Mayo Clin Proc. 2016;91(9):1212-23.

10. Frid AH, Hirsch LJ, Menchior AR, Morel DR, Strauss KW. Worldwide injection technique questionnaire study: injecting complications and the role of the professional. Mayo Clin Proc. 2016;91(9):1224-30.

11. Huang Y, Heng C, Wei J, et al. Influencing factors of glycemic variability in hospitalized type 2 diabetes patients with insulin therapy: a strobe-compliant article. Medicine. (Baltimore). 2017;96(36):e8021.

12. Dagdelen S, Deyneli O, Olgun N, et al. Turkish insulin injection techniques study: complications of injecting insulin among turkish patients with diabetes, education they received, and the role of health care professional as assessed by survey questionnaire. Diabetes Ther. 2018;9(4):1615-28.

13. Di Flaviani A, Picconi F, Di Stefano P, et al. Impact of glycemic and blood pressure variability on surrogate measures of cardiovascular outcomes in type 2 diabetic patients. Diabetes Care. 2011;34(7):1605-9.

14. Esposito K, Nappo F, Marfella R, et al. Inflammatory cytokine concentrations are acutely increased by hyperglycemia in humans: role of oxidative stress. Circulation. 2002;106(16):2067-72.

15. Monnier L, Mas E, Ginet C, et al. Activation of oxidative stress by acute glucose fluctuations compared with sustained chronic hyperglycemia in patients with type 2 diabetes. JAMA. 2006;295(14):1681-7.

16. Frid AH, Kreugel G, Grassi G, et al. New insulin delivery recommendations. Mayo Clin Proc. 2016;91(9):1231-55.

17. Ji J, Lou Q. Insulin pen injection technique survey in patients with type 2 diabetes in mainland China in 2010. Curr Med Res Opin. 2014;30(6):1087-93.

18. Omori K, Kawamura T, Urata M, et al. Effect of recoaching on self-injection of insulin in older diabetic patients-impact of cognitive impairment. Diabetes Res Clin Pract. 2017;130:34-42.

19. Poudel RS, Shrestha S, Piryani RM, Basyal B, Kaucha $\mathrm{K}$, Adhikari S. Assessment of insulin injection practice among diabetes patients in a tertiary healthcare Centre in Nepal: a preliminary study. J Diabetes Res. 2017;2017:8648316.

20. Li FF, Fu LY, Zhang WL, et al. Blood glucose fluctuations in type 2 diabetes patients treated with multiple daily injections. J Diabetes Res. 2016;2016:1028945.

21. Li FF, Xu XH, Fu LY, et al. Influence of acarbose on plasma glucose fluctuations in insulin-treated patients with type 2 diabetes: a pilot study. Int J Endocrinol. 2015;2015:903524.

22. Li FF, Liu BL, Zhu HH, et al. Continuous glucose monitoring in newly diagnosed type 2 diabetes patients reveals a potential risk of hypoglycemia in older men. J Diabetes Res. 2017;2017:2740372.

23. Jehle PM, Micheler C, Jehle DR, Breitig D, Boehm BO. Inadequate suspension of neutral protamine Hagendorn (NPH) insulin in pens. Lancet. 1999;354(9190):1604-7.

24. Brown A, Steel JM, Duncan C, Duncan A, McBain AM. An assessment of the adequacy of suspension of insulin in pen injectors. Diabet Med. 2004;21(6):604-8.

25. Springs MH. Shake, rattle, or roll? Am J Nurs. 1999;99(7):14-6.

26. Nath C. Mixing insulin: shake, rattle, or roll? Nursing. 2002;32(5):10.

27. Lucidi P, Porcellati F, Marinelli Andreoli A, et al. Pharmacokinetics and pharmacodynamics of NPH insulin in type 1 diabetes: the importance of appropriate resuspension before subcutaneous injection. Diabetes Care. 2015;38(12):2204-10.

28. Blanco M, Hernandez MT, Strauss KW, Amaya M. Prevalence and risk factors of lipohypertrophy in 
insulin-injecting patients with diabetes. Diabetes Metab. 2013;39(5):445-53.

29. Kalra S, Mithal A, Sahay R, et al. Indian injection technique study: injecting complications, education, and the health care professional. Diabetes Ther. 2017;8(3):659-72.
30. Wang W, Guo X, Shen G, et al. Skin and subcutaneous tissue thickness at insulin injection sites in Chinese diabetes patients: clinical implications. Diabetes Metab. 2016;42(5):374-7.

31. Ji L, Sun Z, Li Q, et al. Lipohypertrophy in China: prevalence, risk factors, insulin consumption, and clinical impact. Diabetes Technol Ther. 2017;19(1):61-7. 